\title{
A Novel Constitutive Androstane Receptor-Mediated and CYP3A-Independent Pathway of Bile Acid Detoxification
}

\author{
Simrat P. S. Saini, Junichiro Sonoda, Li Xu, David Toma, Hirdesh Uppal, Ying Mu, \\ Songrong Ren, David D. Moore, Ronald M. Evans, and Wen Xie
}

Center for Pharmacogenetics and Department of Pharmaceutical Sciences, University of Pittsburgh, Pittsburgh, Pennsylvania (S.P.S.S, L.X., D.T., H.U., Y.M., S.R. W.X.); Howard Hughes Medical Institute, Gene Expression Laboratory, the Salk Institute for Biological Studies, La Jolla, California (J.S., R.M.E.); and Department of Molecular and Cellular Biology, Baylor College of Medicine, Houston, Texas (D.D.M)

Received August 4, 2003; accepted November 6, 2003

This article is available online at http://molpharm.aspetjournals.org

\begin{abstract}
Cytosolic sulfotransferase (SULT)-mediated sulfation plays an essential role in the detoxification of bile acids and is necessary to avoid pathological conditions, such as cholestasis, liver damage, and colon cancer. In this study, using transgenic mice bearing conditional expression of the activated constitutive androstane receptor (CAR), we demonstrate that activation of CAR is both necessary and sufficient to confer resistance to the hepatotoxicity of lithocholic acid (LCA). Surprisingly, the CARmediated protection is not attributable to the expected and previously characterized CYP3A pathway; rather, it is associated with a robust induction of SULT gene expression and increased LCA sulfation. We have also provided direct evi-
\end{abstract}

dence that CAR regulates SULT expression by binding to the CAR response elements found within the SULT gene promoters. Interestingly, activation of CAR was also associated with an increased expression of the $3^{\prime}$-phosphoadenosine $5^{\prime}$-phosphosulfate synthetase 2 (PAPSS2), an enzyme responsible for generating the sulfate donor $3^{\prime}$-phosphoadenosine-5'-phosphosulfate. Analysis of gene knockout mice revealed that CAR is also indispensable for ligand-dependent activation of SULT and PAPSS2 in vivo. Therefore, we establish an essential and unique role of CAR in controlling the mammalian sulfation system and its implication in the detoxification of bile acids.
Bile acids are end products of cholesterol catabolism that function as both a detergent to solubilize circulating cholesterol remnants and lipophilic vitamins and as a signaling molecule to regulate its own homeostasis. When bound to and activated by bile acids, the farnesoid X receptor (FXR) represses transcription of cholesterol $7 \alpha$-hydroxylase (CYP7A), the rate-limiting enzyme of bile acid synthesis, thereby repressing the conversion of cholesterol to bile acids (Makishima et al., 1999; Parks et al., 1999; Wang et al., 1999). Two other hepatic factors, the small heterodimer partner and liver receptor homolog-1 were subsequently found to be involved in the FXR-mediated CYP7A repression (Goodwin et

This work was supported in part by National Institutes of Health grant R01-ES12479 (to W.X.), the Competitive Medical Research Fund and the Central Research Development Fund from the University of Pittsburgh (to W.X.), and the National Institutes of Health Nuclear Receptor Signaling Atlas orphan receptor program grant U19-DK62434-01 (to R.M.E.). R.M.E. is an Investigator of the Howard Hughes Medical Institute and March of Dimes Chair in Molecular and Developmental Biology. al., 2000; Lu et al., 2000). In addition to their beneficial function, excessive bile acids are potentially toxic when accumulated. For example, the secondary bile acid LCA is a potent cholestatic agent and can cause histological liver damage and other pathological changes unless it is efficiently eliminated (Leuschner et al., 1977). Several lines of evidence have also suggested that toxic bile acids can function as tumor promoters to promote colon cancers (Narisawa et al., 1974).

The efficient detoxification and clearance of bile acids requires the phase I CYP3A enzymes and the phase II cytosolic sulfotransferases (SULTs). The CYP3A enzymes catalyze the hydroxylation of LCA, which promotes LCA elimination (Staudinger et al., 2001; Xie et al., 2001). Recently, the activation of the pregnane $\mathrm{X}$ receptor (PXR) and vitamin $\mathrm{D}$ receptor (VDR) and subsequent induction of CYP3A enzyme has been proposed to be a means to eliminate toxic bile acids (Staudinger et al., 2001; Xie et al., 2001; Makishima et al.,

ABBREVIATIONS: FXR, farnesoid X receptor; LCA, lithocholic acid; SULT, cytosolic sulfotransferase; PXR, pregnane X receptor; CAR, constitutive androstane receptor; VDR, vitamin D receptor; PAPS, 3'-phosphoadenosine-5'-phosphosulfate; PB, phenobarbital; TCPOBOP, 1,4-bis[2(3,5-dichloropyridyloxy)]benzene; PAPSS2, 3'-phosphoadenosine 5'-phosphosulfate synthetase 2; VP, virus protein; EMSA, electrophoretic mobility shift assay; RXR, retinoid X receptor; DHEA, dehydroepiandrosterone; TetRE, tetracycline responsive element; tTA, tetracyclineresponsive transcriptional activator; Dox, doxycycline; GAPDH, glyceraldehyde-3-phosphate dehydrogenase. 
2002). The phase II SULTs are also important for bile acid detoxification. SULTs catalyze the transfer of a sulfonyl group from the cosubstrate 3 '-phosphoadenosine-5'-phosphosulfate (PAPS) to the acceptor substrates to form sulfate or sulfamate conjugates. LCA is a preferred substrate for SULT2A9/hydroxysteroid sulfotransferase (Chen and Segel, 1985; Radominska et al., 1990; Song et al., 2001). The sulfated LCA shows less cytotoxicity than LCA when exposed to cells or animals (Leuschner et al., 1977). Although SULTs play a key role in a number of critical biological pathways, little is known about the regulatory pathways that control SULT gene expression.

The orphan nuclear constitutive androstane receptor (CAR) was first shown to function as a xenobiotic receptor by activating the CYP2B genes. Subsequently, this activation was found to be potentiated by phenobarbital (PB) and TCPOBOP (Honkakoski et al., 1998; Tzameli et al., 2000; Xie et al., 2000a), and the latter was identified as a CAR agonistic ligand. Response to both inducers was completely lost in CAR knockout mice (Wei et al., 2000). CAR was later shown to cross-regulate CYP3A genes in cell cultures by sharing the previously identified PXR response elements (Xie et al., 2000b; Goodwin et al., 2001; Wei et al., 2002). CAR was more recently implicated in transactivating genes that encode the phase II UDP-glucuronosyltransferase 1A1 (Sugatani et al., 2001; Huang et al., 2003; Xie et al., 2003) and the drug transporter multidrug resistance-associated protein 2 (MRP2) (Kast et al., 2002). Although CAR has been well characterized as a cytochrome $\mathrm{P} 450$ gene regulator, no direct evidence that relates the function of this xenobiotic receptor to the transcriptional activation of SULT is available. PB and TCPOBOP have been shown to induce members of the SULT family, although the molecular basis remains to be defined (Runge-Morris et al., 1999; Garcia-Allan et al., 2000; Maglich et al., 2002). Both PB and TCPOBOP are efficacious CAR activators, suggesting this as a plausible signaling pathway for SULT transcription. The SULT induction by TCPOBOP is apparently CAR-dependent (Maglich et al., 2002), but a DNA microarray analysis with PB-treated mice failed to identify SULT as a target (Ueda et al., 2002).

In this report, we show that activation of CAR in transgenic mice confers resistance to the hepatotoxicity of LCA. The protection is not caused by CYP3A; instead, it is associated with the induction of both SULTs and PAPS synthetase 2 (PAPSS2), an enzyme responsible for generating the cosubstrate PAPS. Moreover, CAR is indispensable for SULT and PAPSS2 induction by PB and TCPOBOP. We propose that activation of CAR facilitates bile acid detoxification via a combined induction of the sulfation system.

\section{Materials and Methods}

Animals, Drug Treatment, and Histology Evaluation. The creation of PXR and CAR null mice has been described before (Wei et al., 2000; Xie et al., 2000b). The PXR/CAR double-knockout mice were created by cross-breeding. When necessary, mice were subjected to a single intraperitoneal injection of $\mathrm{PB}(40 \mathrm{mg} / \mathrm{kg})$ or TCPOBOP (3 mg/kg) $24 \mathrm{~h}$ before sacrifice. To generate the tetracycline responsive element (TetRE)VP-CAR transgene, VP-CAR cDNA was excised from pCMX-VP-mCAR (Xie et al., 2000a), and cloned into the TetRE transgene cassette (Xie et al., 1999). The Lap-tTA mice were obtained from The Jackson Laboratory (Bar Harbor, ME). When necessary, doxycycline (Dox; Sigma, St. Louis, MO) was di- luted in $5 \%$ sucrose in water to a final concentration of $2 \mathrm{mg} / \mathrm{ml}$ and supplied as drinking water. The Dox-laced water was changed every 2 to 3 days. For LCA treatment, mice were given daily treatments of LCA ( $8 \mathrm{mg} /$ day) or vehicle via gavage and were sacrificed $24 \mathrm{~h}$ after the last treatment (Xie et al., 2001). For histology evaluation, tissues were fixed in $4 \%$ formaldehyde, embedded in paraffin, sectioned at 5 $\mu \mathrm{m}$, and stained for hematoxylin and eosin. The use of mice in this study has complied with all relevant federal guidelines and institutional policies.

DNA-Binding Analysis. Electrophoretic mobility shift assays (EMSA) were performed using in vitro-transcribed and -translated proteins (TNT; Promega, Madison, WI) as described previously (Xie et al., 2000a). Oligonucleotides used were: rat SULT2A1/IR0, 5'TTTGGGGGTCATGAACTTGGGC-3'; mouse SULT2A9/IR0, 5'-TTTGG GGGTAATGAACT TGGGC-3'; and SULT/IR0 mut, 5'-TTTGGGGGTACCGAACTTGGGC-3'.

Plasmid Constructs and Transfection. The synthetic reporter thymidine kinase (tk)-IR0-Luc, the natural promoter reporter pGLSULT, and their mutant variants were described before (Sonoda et al., 2002). The expression vectors for mCAR, hPXR, mPXR, and hRXR $\alpha$ were as described previously (Xie et al., 2000a,b). CV-1 and HepG2 cells were transfected in 48-well plates using $N$-[1-(2,3-dioleoyloxy)propyl]- $N, N, N$-trimethylammonium methylsulfate (Roche, Indianapolis, IN) and LipofectAMINE (Invitrogen, Carlsbad, CA), respectively. When necessary, cells were treated with androstenol (5 $\mu \mathrm{M})$, TCPOBOP $(250 \mathrm{nM})$, pregnenolone-16 $\alpha$-carbonitrile $(10 \mu \mathrm{M})$, and St. John's wort $(300 \mu \mathrm{g} / \mathrm{ml})$ in media containing $10 \%$ charcoalstripped serum. The transfection efficiency was normalized against the $\beta$-galactosidase activities from the cotransfected CMX- $\beta$-galactosidase vector.

Northern Blot Analysis. Total RNAs were prepared from tissues using the TRIzol Reagent (Invitrogen). Northern hybridization was carried out as described previously (Xie et al., 2000b). The cDNA probes for SULTs, PAPSS2, CYP3A11, and CYP2B have been described previously (Xie et al., 2000a, b; Sonoda et al., 2002).

Sulfotransferase Assay. Sulfotransferase assay was carried out using $\left[{ }^{35} \mathrm{~S}\right]$ PAPS (PerkinElmer Life and Analytical Sciences, Boston, MA) as described previously (Sonoda et al., 2002). In brief, 5 to 10 $\mu \mathrm{g} / \mathrm{ml}$ total liver cytosolic extract was used with $2 \mu \mathrm{M}$ LCA, $5 \mu \mathrm{M}$ dehydroepiandrosterone (DHEA), or $4 \mu \mathrm{M} p$-nitrophenol as substrate. After the reactions, free $\left[{ }^{35} \mathrm{~S}\right] \mathrm{PAPS}$ was removed by extracting with ethyl acetate. The aqueous phase was then measured in a liquid scintillation counter for radioactivity. Control reactions that do not contain substrate were also carried out in parallel, and their radioactivity was subtracted from test reactions. Two to four pairs of mice were used for each SULT assay, and each reaction was run in triplicate.

\section{Results}

Conditional Expression of the Activated CAR in Transgenic Mice. To examine the effects of CAR activation in xenobiotic regulation, we created a transgenic mouse system that allowed conditional expression of a constitutively activated CAR (VP-CAR) (Xie et al., 2000a) in the liver. Two lineages of transgenic mice were used as diagramed in Fig. 1A. First, we created the TetRE-VP-CAR transgene that encodes VP-CAR under the control of a minimal cytomegalovirus promoter and the TetRE (Fig. 1A). The TetRE-VPCAR mice were subsequently bred with the Lap-tTA activator line to generate bi-transgenic animals. Driven by the liver-specific Lap (CCAAT/enhancer-binding protein- $\beta$ ) promoter, the Lap-tTA transgene directed the expression of the tetracycline-responsive transcriptional activator (tTA) constitutively and exclusively in the hepatocytes (Kistner et al., 1996). We anticipated that tTA bound to TetRE and conse- 
quently induced the expression of VP-CAR only in the absence of Dox. Addition of Dox will result in the displacement of tTA from TetRE and will silence VP-CAR expression (TetOff).

Transgene expression was assessed by Northern blot analysis of liver RNA using the tTA cDNA probe. Because tTA contains the VP16 activation domain that is also present in VP-CAR, this probe recognizes mRNA of both tTA and VPCAR. Similar levels of tTA expression were detected in the livers of all Lap-tTA transgenic mice whether they harbored this transgene alone or in combination with the TetRE-VPCAR transgene (Fig. 1B). Moreover, expression of the LaptTA mRNA was independent of Dox treatment (Fig. 1B, compare lanes 4 and 5). Two tTA-specific transcripts were detected, consistent with our previous observation (Xie et al., 1999). No VP-CAR expression was detected in the TetRE-VPCAR single transgenic mice (Fig. 1B, lane 2). In contrast, a robust expression of the 2.5-kilobase VP-CAR was achieved in the bitransgenic animal in the absence of Dox (Fig. 1B, lane 4). Moreover, the expression of CYP2B10, a known CAR target gene, was also markedly induced in the VP-CARexpressing livers. As expected, the administration of Dox in drinking water in bitransgenics resulted in the silencing of

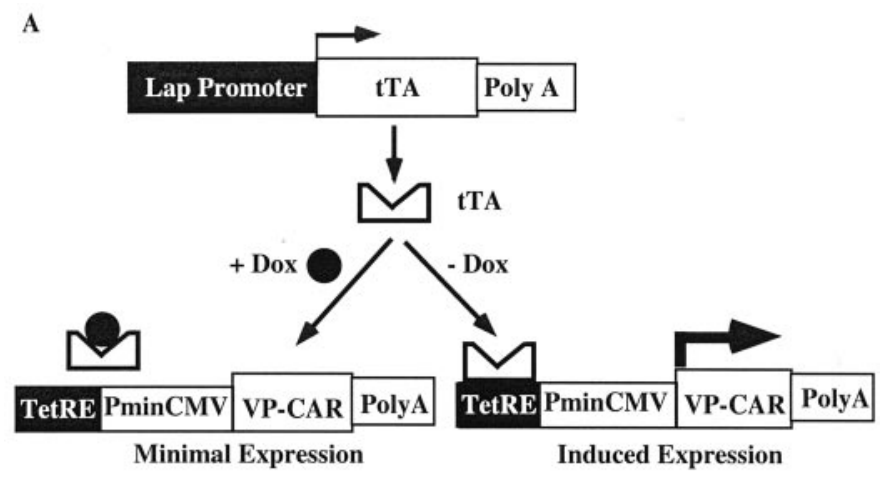

B

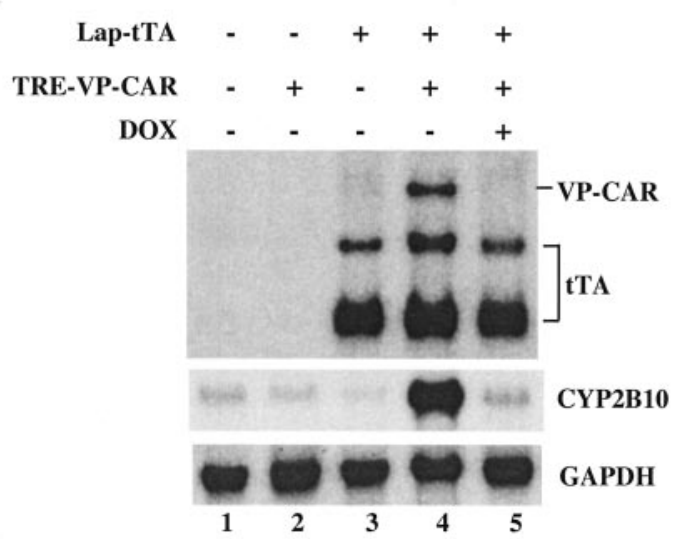

Fig. 1. Creation of transgenic mice that harbor conditional expression of the activated CAR in the liver. A, a schematic outline of the Lap-tTA/ TetRE-VP-CAR two-component Tet-Off transgenic system. The Lap-tTA transgene directs the expression of the tTA activator to the liver. The binding of tTA to the TetRE and the induction of the transgene VP-CAR should only occur in the absence of Dox. B, liver-specific conditional expression of VP-CAR. Liver RNAs of mice with indicated genotypes were subjected to Northern blot analysis. The mouse in lane 5 was subjected to 5 days of Dox treatment. The membrane was hybridized with the tTA probe that recognizes both tTA and VP-CAR transcripts. The membrane was subsequently stripped and reprobed with CYP2B10, with GAPDH as a loading control. both VP-CAR expression and CYP2B10 induction (Fig. 1B, lane 5). Therefore, the expression of VP-CAR in the liver was completely reversible upon Dox treatment. The expression of VP-CAR was restricted to the liver. No VP-CAR transcripts were detected in the intestine, and tTA was not expressed in the intestine (data not shown). The hepatic expression of the endogenous mouse CAR remained unchanged in the presence of VP-CAR (data not shown). Because the presence of single transgene did not cause VP-CAR expression and had no effect on the expression of a number of known CAR targets genes that we examined (Fig. 1B; data not shown), both the single transgenic and wild-type mice were used as control animals for the bitransgenic mice in the following animal experiments.

Activation of CAR Confers Resistance to LCA Hepatotoxicity. The secondary bile acid LCA is toxic and known to cause cholestasis and associated hepatotoxicity (Staudinger et al., 2001; Xie et al., 2001). To examine whether or not activation of CAR had effects on LCA sensitivity, adult bitransgenic mice or control littermates were dosed with vehicle solvent or LCA for 4 days before liver histological evaluation. As expected, the wild-type liver exhibited areas of necrosis after LCA exposure (Fig. 2B), consistent with our previous report (Xie et al., 2001). In a sharp contrast, the liver of bi-transgenic mice showed virtually no histological changes upon LCA treatment (Fig. 2C). The absence of induced pathology in the bi-transgenic mice demonstrates that sustained activation of CAR is sufficient to prevent LCA-mediated histological liver damage. This xenoprotection is CAR activation-dependent, because treatment of Dox blocked protection (Fig. 2D). As expected, Dox treatment alone had no effect on liver histology of the wild-type mice (data not shown), and the Dox-treated wild-type animals remained sensitive to LCA (Fig. 2E).

Activation of CAR Induces SULTs and PAPSS2 in Transgenic Mice. To delineate the molecular mechanism of LCA resistance, we profiled the expression of hepatic genes encoding bile acid-detoxifying enzymes in the VP-CAR mice. The phase I CYP3A11, a primary PXR target gene, has been shown to be cross-regulated by CAR in cell cultures (Xie et al., 2000a). Much to our surprise, the expression of CYP3A11 mRNA was slightly suppressed rather than induced in the bitransgenic mice (Fig. 3A). A CYP3A enzymatic assay using testosterone as a substrate also confirmed the absence of CYP3A induction in VP-CAR mice (data not shown). In contrast, the expression of the phase II SULT 2A9 mRNA was markedly induced in the VP-CAR mice (Fig. 3A). The induction was seen in both sexes, although the female mice had a higher basal level of this isoform (Klaassen et al., 1998). The sustained induction of SULT2A9 was VP-CAR dependent, because treatment with Dox for 7 days resulted in complete loss of 2A9 induction in both male and female mice, presumably because of the absence of VP-CAR expression (Fig. 1B). As expected, Dox treatment has no effect on the expression either SULT2A9 or CYP3A11 in the control mice (Fig. 3A). Of note was that the expression of CYP3A11 in the VP-CAR mice remained inducible in response to TOPOBOP (Fig. 3B), suggesting that the lack of CYP3A11 induction in the unchallenged mice was not caused by the unresponsiveness of CYP3A11 in this transgenic line.

We also analyzed the expression of several other SULT isoforms. The expression of SULT1A4 was increased in the 
bitransgenic mice, whereas the expression of SULT1D1 remained unchanged (Fig. 3C). Thus, the regulation of SULT by CAR seemed to be isoform-specific. The sulfation reaction requires the donation of a sulfonyl group from the cosub- strate PAPS. Surprisingly, the expression of hepatic PAPSS2, the enzyme that catalyzes the formation of PAPS from inorganic sulfate (Lyle et al., 1994), was also elevated in the bitransgenic mice (Fig. 3D, lane 2). The PAPSS2 induc-
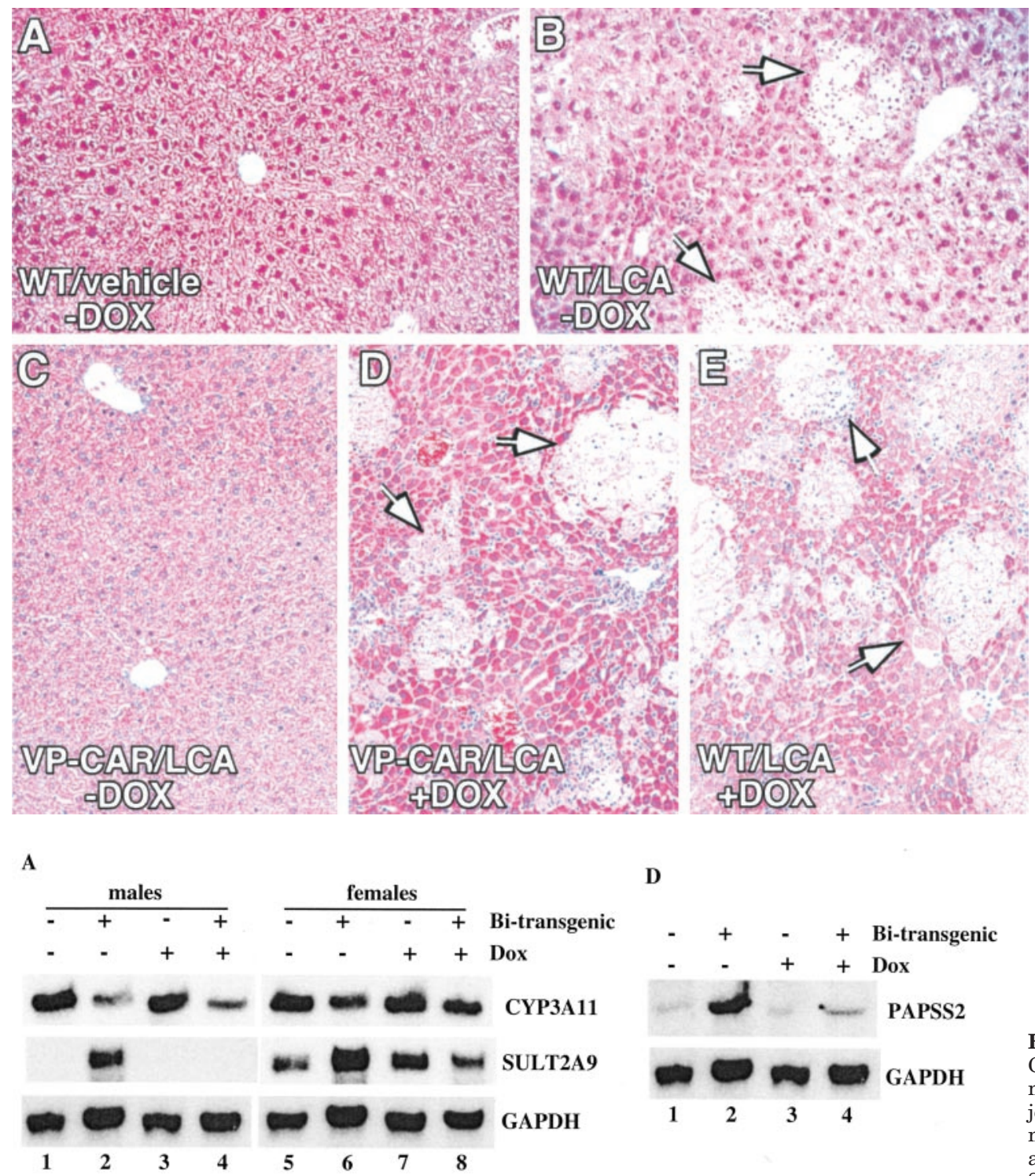

A

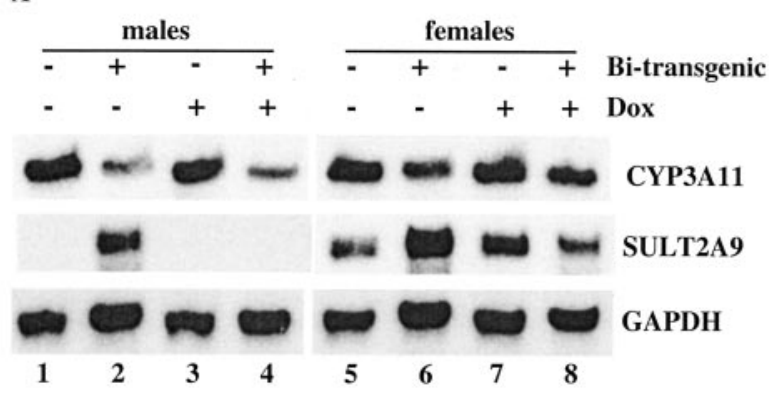

D

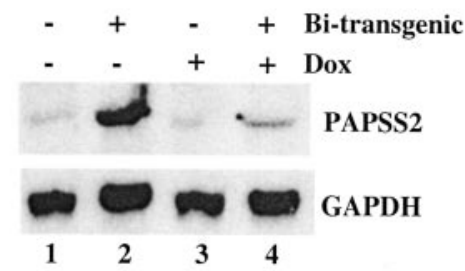

B

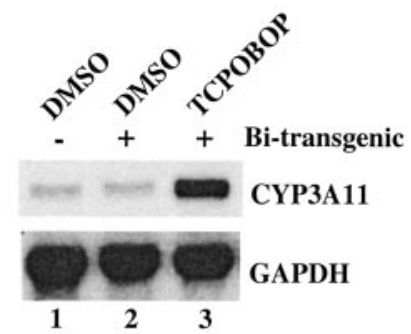

C

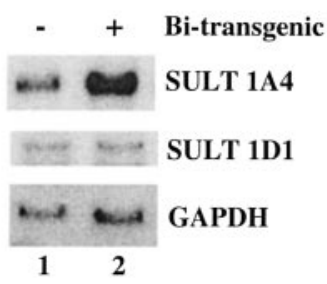

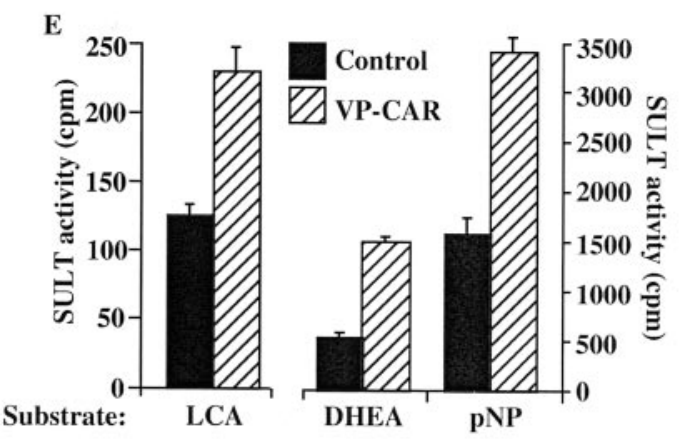

Fig. 3. The expression of SULTs, but not CYP3A11, is induced in the VP-CAR mice. A-C, total liver RNAs were subjected to Northern blot analysis. The membranes were probed for CYP3A11 and SULT2A9 (A), CYP3A11 (B), SULT1A4 and 1D1 (C), and PAPSS2 (D). Mice in lanes 3, 4, 7, and 8 of $\mathrm{A}$ and lanes 3 and 4 of D were treated with Dox for 7 days before tissue harvest. GAPDH probing was used as loading control. E, increased SULT activity in VP-CAR mice. Cytosolic liver extracts from bitransgenic male mice or control littermates were subjected to sulfation assay using the substrates of LCA, DHEA, and $p$-nitrophenol. $\left[{ }^{35}\right.$ S]PAPS was used the sulfate donor. Radioactivity was measured by scintillation counter after separation and removal of free $\left[{ }^{35} \mathrm{~S}\right] \mathrm{PAPS}$. Results represent the averages and standard error. 
tion was lost upon 7 days of Dox treatment (Fig. 3D, lane 4). Our data suggest that CAR may function as a global regulator of sulfation cascade by controlling the production of both SULT enzymes and the cosubstrate PAPS.

The increased expression of SULTs was also reflected at enzymatic levels. SULT2A9 is known to sulfonate bile acids such as LCA, steroid hormones and their precursors, such as DHEA, and carcinogenic xenobiotics, such as $p$-nitrophenol. Compared with the control mice, the bitransgenic animals exhibited about 2-fold higher hepatic sulfation activity toward LCA (Fig. 3E). The sulfation of DHEA and $p$-nitrophenol was also more than doubled (Fig. 3E). Together, these results suggest that members of the cytosolic SULT family are under the positive control of CAR.

Identification of CAR Binding Sites within the SULT Gene Promoters. To understand the underlying mechanism of $S U L T$ regulation by CAR, we went on to analyze the $5^{\prime}$ flanking region of SULT genes. Sequence analysis of the rodent SULT2A gene promoters revealed an IR-0 (inverted repeats without a spacing nucleotide) type of nuclear receptor response element (Fig. 4A) (Runge-Morris et al., 1999; Song et al., 2001; Sonoda et al., 2002). The rat 2A1/IR0 element was shown to bind to and mediate the transactivation by PXR (Sonoda et al., 2002) and FXR (Song et al., 2001).

A

Rat SULT2A1/IR0 (nt -192) tgg GGGTCATGAACT tgg (nt -175)

Mouse SULT2A9/IR0 (nt -192) ttg GGGTAATGAACT tgg (nt -175)

SULT/IR0 mut ttg GGGTACCGAACT tgg

B

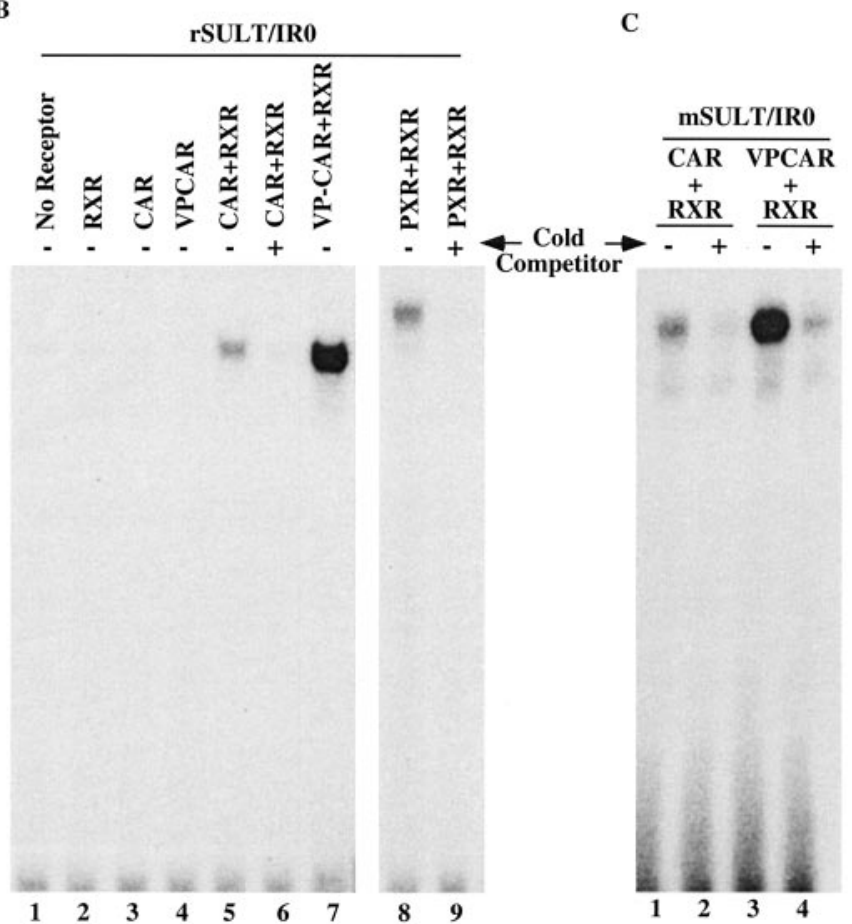

Fig. 4. Identification of CAR binding sites in the rodent SULT gene promoters. A, the partial DNA sequence of the rat SULT2A1 and mouse SULT2A9 gene promoters. The IR0 elements are boxed. A mutant variant was also shown with the mutated nucleotides underlined. B and C, $\mathrm{CAR} / \mathrm{RXR} \alpha$ or VP-CAR/RXR $\alpha$ heterodimers bound to the IR0. EMSA was performed using in vitro-synthesized receptor proteins and radiolabeled oligonucleotides of rat (B) or mouse (C) IR0. The binding of PXR/RXR $\alpha$ was included as a positive control.
We examined whether this IR0 element can also bind to CAR. EMSAs were used to determine the ability of CAR to bind to SULT/IR0 using in vitro-synthesized receptor proteins and ${ }^{32}$ P-labeled oligonucleotide probe. As shown in Fig. 4B, both the wild-type CAR and its activated variant VP-CAR bound the rat SULT2A1/IR0 efficiently (Fig. 4B, lanes 5 and 7). The binding was dependent on the presence of their obligatory heterodimerization partner RXR; no DNA binding was seen in the absence of RXR (Fig. 4B, lanes 3 and 4). These results demonstrate that CAR/RXR or VP-CAR/RXR binds SULT/ IR0 in a fashion similar to the binding of PXR/RXR to the same element (Fig. 4B, lane 8). This represents another example of the sharing of binding sites by xenobiotic nuclear receptors (Xie et al., 2000a). The integrity of this IR0 element is essential for the binding, because the binding was abrogated when the IR0 was disrupted by mutation (data not shown). The binding of IR0 by CAR was also specific, inasmuch as efficient competition of binding was achieved by excess unlabeled wild-type IR0 (Fig. 4B, lane 6). Specific binding of both CAR and VP-CAR to the conserved mouse SULT2A9/IR0 was also observed (Fig. 4C). Whereas the VPCAR exhibited a binding specificity similar to that of its wild-type counterpart, VP-CAR seemed to have higher affinity toward the IR0 elements (Fig. 4, B and C).

CAR Activates SULTs in Cultured Cells. Transfectionbased assays were used to determine whether CAR can transactivate SULT by binding to the IR0 elements in cultured cells. First, luciferase reporter genes, containing the wild-type rat and mouse IR0 or their mutant variant upstream of a minimal tk promoter, were constructed and transfected into CV-1 cells together with expression vectors for mouse CAR or PXR receptor in the presence of RXR. A panel of mCAR agonist and inverse agonist compounds was tested. As shown in Fig. 5A, reporter genes derived from both rat and mouse SULT2A genes were activated by CAR in the absence of ligand. The activation was substantially inhibited by the inverse agonist androstenol but modestly potentiated by the agonist TCPOBOP. The agonistic effect of TCPOBOP was better manifested by its ability to reverse the inhibitory effect of androstenol when both ligands were added simultaneously, consistent with previously reports (Honkakoski et al., 1998; Tzameli et al., 2000 et al., Xie et al., 2000b). As expected, PXR also activated the same reporter genes (Sonoda et al., 2002) but with a distinctive ligand profile (Fig. 5B). For example, androstenol only showed marginal effect, whereas TCPOBOP is completely ineffective on PXR. In contrast, St. John's wort, an herbal antidepressant and PXR activator, induced SULT reporter genes by activating PXR (Fig. 5B) but not CAR (data not shown). Consistent with DNA binding results, the activation by CAR or PXR was abrogated when the IR0 was disrupted (Fig. 5, A and B). Thus, the IR0 sites are mediators for both the binding and activation of SULT2A by CAR.

The activation of SULT2A by the wild-type or constitutively activated CAR was also seen when a luciferase reporter that contains the natural promoter of rat SULT2A1 gene (nucleotides -1023 to +38 ) was used. The reporter, PGL-SULT, was cotransfected with the wild-type or activated mCAR into human hepatoma HepG2 cells or primary rat hepatocytes followed by ligand treatment. HepG2 or hepatocytes were used because this promoter was not responsive in non-hepatocyte-derived cells (data not shown). Con- 
sistent with the observations in $\mathrm{CV}-1$ cells, activation of the natural SULT promoter by CAR in HepG2 cells was inhibited by androstenol. TCPOBOP not only activated CAR by itself but also reversed the inhibitory effect of androstenol (Fig. 5C, lane 2). Interestingly, although VP-CAR exhibited significantly higher constitutive activity, it was also subjected to ligand effects similar to those of its wild-type counterpart (Fig. 5C, lane 3). The activation by CAR and VP-CAR was abolished when a promoter variant that contains the mutant IR0 was cotransfected (lanes 4 and 5).

Car Is Indispensable for Ligand-Dependent Activation of SULT. The expression of rodent SULT has been shown to be induced by $\mathrm{PB}$ and TCPOBOP, two reported CAR agonists (Runge-Morris et al., 1999; Garcia-Allan et al., 2000; Maglich et al., 2002). Having established that activation of CAR is sufficient to induce SULT2A9, we went on to examine whether xenobiotic receptors, such as CAR and PXR, are necessary for the ligand-dependent activation of SULT. We applied a single dose of PB and TCPOBOP to wild-type, PXR-null (Xie et al., 2000b), CAR-null (Wei et al., 2000), and PXR/CAR double-knockout mice. The double-knockout mice were created by cross-breeding, and the absence of both PXR and CAR mRNA was confirmed by Northern blot analysis (Fig. 6A). Livers were harvested $24 \mathrm{~h}$ after treatment, and the expression of SULT was evaluated by Northern blot analysis. As shown in Fig. 6B, the expression of both SULT2A9 and PAPSS2 was induced by either PB or TCPOBOP, as expected. Both the basal and PB- and TCPOBOP-inducible expression of SULT2A9 and PAPSS2 was sustained in the PXR-null mice, suggesting that PXR was dispensable for this induction. In contrast, disruption of the CAR locus led to a loss of SULT2A9 and PAPSS2 induction by TCPOBOP (Fig. 6C) and PB (data not shown) in both CAR-null and CAR/PXR double-knockout backgrounds. Together, our results demonstrate that CAR, but not PXR, is the bona fide receptor to mediate SULT and PAPSS2 induction by $\mathrm{PB}$ and TCPOBOP in vivo.

\section{Discussion}

Activation of PXR and VDR have been implicated in bile acid detoxification by inducing CYP3A (Staudinger et al., 2001; Xie et al., 2001; Makishima et al., 2002), but little is known about whether CAR also plays a role in bile acid detoxification. In this report, we show that the activation of CAR is both necessary and sufficient to confer resistant to the hepatotoxic LCA. Unexpectedly, the protection is CYP3A-
A

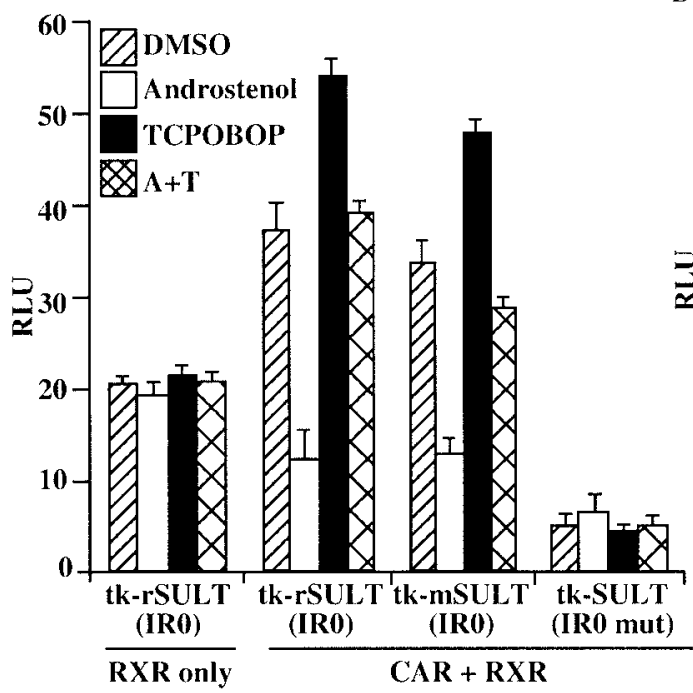

B
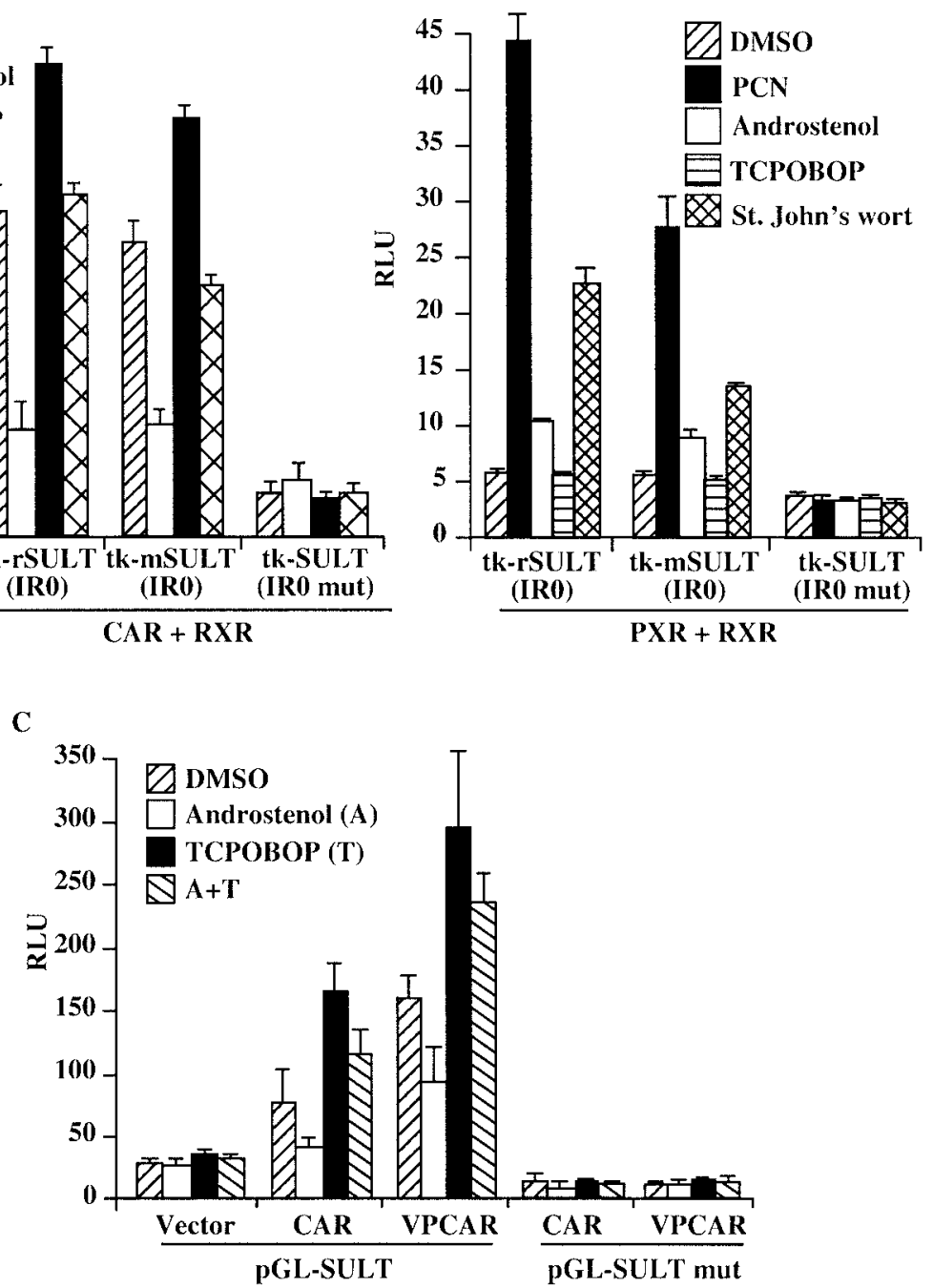

Fig. 5. CAR activates $S U L T$ gene expression in cell cultures. A, the synthetic tkSULT/IR0-Luc reporters or their mutant variants were transfected into $\mathrm{CV}-1$ cells in the presence of expression vectors for CAR and RXR $\alpha$. Cells were subsequently treated with individual or combination of compounds. Results shown are normalized relative luciferase units and represent the averages and standard error from triplicate assays. B, similar transfections but using PXR receptor. C, CARmediated and IR0-dependent activation of the natural rat SULT2A1 gene promoter. The natural SULT promoter or its mutant variant were transfected into HepG2 cells in the presence of expression vectors for CAR or VP-CAR. Cells were subsequently mock-treated or treated with indicated compounds. Ligand concentrations: androstenol, $5 \mu \mathrm{M}$; TCPOBOP, $250 \mathrm{nM}$; pregnenolone-16 $\alpha$ carbonitrile (PCN), $10 \mu \mathrm{M}$; St. John's wort, $300 \mu \mathrm{g} / \mathrm{ml}$. 
independent, but can be explained, at least in part, by the ability of CAR to activate the detoxifying sulfonation system.

The identification of SULTs as targets of CAR has implications in bile acid detoxification, drug metabolism, and carcinogenesis. Sulfation is an essential step in the detoxification of bile acids and is necessary to avoid pathologic conditions, such as cholestasis, liver damage, and colon cancer (Fisher et al., 1971; Narisawa et al., 1974; Leuschner et al., 1977). The protection against LCA toxicity in VP-CAR mice suggests a potential therapeutic strategy for the design of CAR agonists to target cholestasis and to prevent colon

A

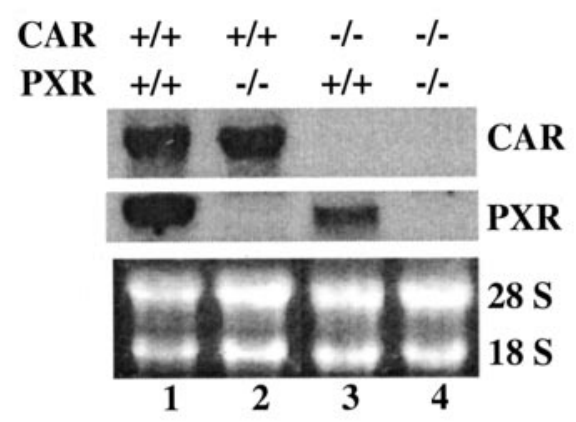

B
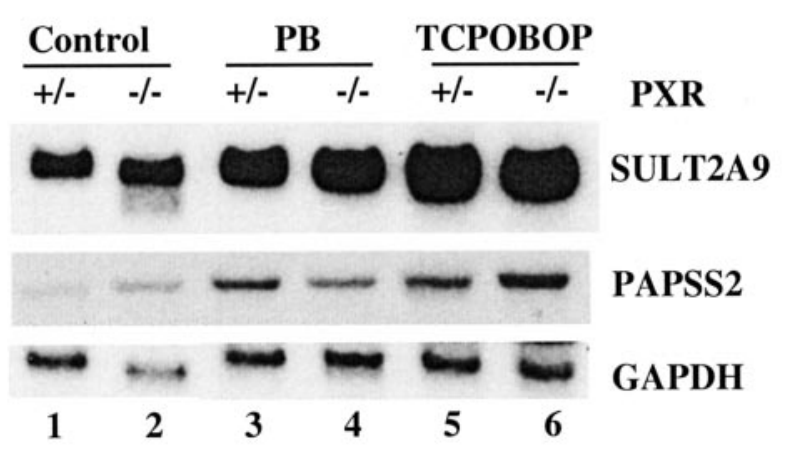

C

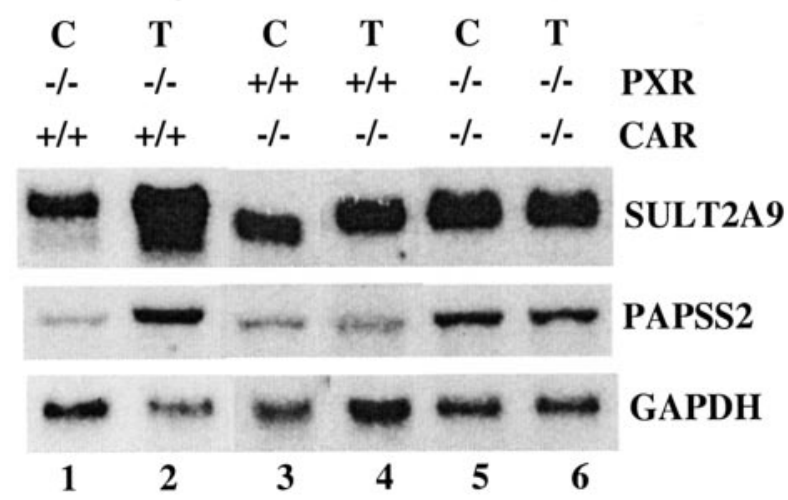

Fig. 6. CAR, but not PXR, is essential for the induction of SULT and PAPSS2 in vivo. A, the creation of PXR/CAR double-knockout mice. The absence of both PXR and CAR mRNA was confirmed by Northern blot analysis. An ethidium bromide staining of the agarose gel was shown to indicate an equal loading. $\mathrm{B}$, The $\mathrm{PXR}^{+-}$or PXR-null mice were subjected to a single intraperitoneal injection of solvent (lanes 1 and 2), PB (lanes 3 and 4), or TCPOBOP (lanes 5 and 6). Total liver RNAs were subject to Northern blot analysis. The membranes were first probed for SULT2A9 and PAPSS2 and subsequently stripped and reprobed for GAPDH as a loading control. The PXR ${ }^{+/-}$mice had the same SULT2A9 basal expression and PB- and TCPOBOP-inducibility as their wild type littermates (data not shown). C, regulation of SULT2A9 and PAPSS2 in PXR-null (lanes 1 and 2), CAR-null (lanes 3 and 4) and CAR/PXR doubleknockout (lanes 5 and 6) mice. C, vehicle control; T, TCPOBOP. cancer. We have previously shown that the PXR- and VDRmediated CYP3A induction was also important for LCA clearance (Xie et al., 2001; Makishima et al., 2002). However, the resistance to LCA toxicity in the VP-CAR mice was clearly CYP3A-independent, because this enzyme was not induced in the VP-CAR mice (Fig. 3A). The attribution of SULT2A9 induction to the LCA resistance in VP-CAR mice is also consistent with a recent report that SULT2A9/hydroxysteroid sulfotransferase-mediated LCA sulfation was a major pathway for protection against LCA-induced hepatotoxicity. The FXR-null female mice exhibited enhanced resistance to LCA, which was associated with significantly increased hepatic SULT2A expression and LCA sulfation (Kitada et al., 2003). The excretion and elimination of bile acids are also facilitated by the UDP-glucuronosyltransferase (Radominska et al., 1990) and the canalicular bile acid transporter MRP2 (Kullak-Ublick et al., 2000); both are known CAR target genes (Sugatani et al., 2001; Kast et al., 2002; Huang et al., 2003; Xie et al., 2003), so we can not exclude the possibility that additional elements of the bile acid detoxifying system also contribute to the protection.

Sulfation by SULTs is known to play a critical role in the metabolism of many drugs, including the most commonly used anti-inflammatory agent, acetaminophen (Tylenol), whose overdoses are among the leading causes for clinical acute liver failure. Zhang et al. (2002) recently identified CAR as a key regulator of acetaminophen metabolism and hepatotoxicity. CAR activators induced the expression of several acetaminophen-metabolizing enzymes, including the glutathione $S$-transferase $\mathrm{Pi}$, a phase II enzyme that inactivates the toxic acetaminophen quinone metabolite (Zhang et al., 2002). Our results suggest that the induction of SULTs may also contribute to the CAR-mediated xenobiotic response in our body's handling of acetaminophen exposure.

It is known that inherited differences in the enzymatic activity of sulfotransferases are likely to influence cancer risk. For example, several studies have shown that estrogen receptor-positive breast cancer cells have very low estrogen sulfotransferase activity (Falany and Falany, 1996). In addition to sulfonating the growth-promoting steroid hormones, SULTs have been shown to catalyze the sulfation of a wide array of chemical carcinogens. Indeed, sulfation of the carcinogenic $p$-nitrophenol is markedly increased in VP-CAR mice (Fig. 3E). Thus, the creation of these transgenic mice not only demonstrates a role for CAR in SULT regulation but also provides a potential in vivo model to assess the molecular dynamics of carcinogenesis and the contribution of sulfation to this process. Although sulfation typically leads to detoxification, certain xenobiotics can become mutagenic once sulfonated (Glatt, 1997).

We used the unique VP-CAR transgenic system to identify and characterize the cytosolic SULTs as targets of CAR. Ligand-facilitated target gene identification using wild-type or gene knockout mice has been widely used (Maglich et al., 2002; Ueda et al., 2002). We consider the use of the VP-fusion receptor transgenes to have unique advantages over drug treatment. This is particularly important because we now know that treatments with receptor pan-agonists, such as bile acids, may affect multiple receptors depending upon the tissue context (Staudinger et al., 2001; Xie et al., 2001; Makishima et al., 2002). Moreover, several lines of evidence suggest that ligand treatment may have additional transcrip- 
tional consequences independent of the presence of endogenous receptor. For example, Ueda et al. identified 168 differentially expressed tags in response to PB treatment. However, nearly half of these tags were similarly affected in the CAR knockout mice (Ueda et al., 2002). Bypassing the requirement of ligand treatment, the VP fusion of receptors provides a unique strategy not only to study the biological consequences of receptor activation but also to identify target genes (Rosenfeld et al., 2003). The utility and practicality of this strategy have been proven in our previous creation and characterization of the Alb-VP-hPXR (previously known as VPSXR) transgenic mice, in which the activated hPXR was expressed in the liver (Xie et al., 2000b). Even though the VP fusion receptor of CAR represents a unique tool to genetically dissect the gene regulation by CAR, we recognize that the level of CAR expression and/or activity in the VP-CAR mice may be substantially higher than the endogenous CAR activity in response to endogenous ligands in normal physiology. However, the limitation of this genetic model does not exclude the potential that pharmacological modulation of CAR activity may be applied to detoxify bile acids.

In addition to CAR, several other orphan receptors have also been implicated in the regulation of $S U L T$ gene expression. For example, we have recently reported the DHEA SULT as a direct transcriptional target of PXR in response to bile acids and many other PXR ligands (Sonoda et al., 2002). FXR, a prototypic bile acid receptor, was also shown to regulate DHEA SULT in cultured cells (Song et al., 2001). Thus, three distinct nuclear receptors, CAR, PXR, and FXR, may collaborate to regulate the sulfation cascade to detoxify xenoand endotoxins. Interestingly, all three receptors use the same IR0 response elements found in SULT2A gene promoters. This represents another example of the sharing of binding sites by xenobiotic nuclear receptors, the underlying mechanism of the proposed "fail-safe pathways" in xenobiotic regulation (Xie et al., 2000a). Intriguingly, although CAR is both necessary and sufficient for SULT induction, loss of CAR and PXR individually or in combination does not suppress the basal expression of SULT (data not shown). It is possible that the sustained basal expression of SULT2A9 in CAR/PXR double-knockout mice is mediated, at least in part, by FXR.

Last but not least, the Tet-Off transgenic system has many attributes to facilitate the study of nuclear receptor functions in vivo. Because of their critical roles in development and normal physiology, embryonic and perinatal lethality is not unusual when genes encoding nuclear receptors or its genetic variants were disrupted via homologous recombination or overexpressed through transgenes. The concept of conditional expression was conceived to overcome the many potential circumstances of embryonic and perinatal lethality that accompany changes in the expression of many important genes (Xie et al., 1999). Tetracycline/doxycycline regulated systems seem to provide a solution. As to the study of xenobiotic receptors, not only can the application of inducible systems overcome potential lethality but it can also effectively establish the role of specific xenobiotic receptors in drug metabolism, drug-drug interactions, and drug toxicity. For example, the reversibility of VP-CAR expression and the resultant SULT induction and protection against xenotoxicants can be applied to study the effect of SULT activity on xenobiotic clearance in a developmental stage- and chemical exposure-specific manner.

Although CAR itself exhibits certain levels of constitutive activity, the VP-CAR is fundamentally different in that the VP-CAR seems to bypass the biological system by directly transactivating genes in the nucleus, whereas the wild-type CAR normally resides in the cytoplasm. Compared with PXR, although CAR is called a "xenobiotic receptor", it actually does not bind most of the ligands that activate it. Thus, CAR may function as a sensor for the class of xenobiotic compounds that act through a cell surface pathway to trigger CAR translocation from the cytosol to the nucleus. PXR directly binds bile acids but CAR does not. Therefore, we believe that the ability of CAR to control bile acid homeostasis is more than a simple extension of the PXR function. The development of inducible VP-CAR transgenic system provides a unique approach to further dissect the CAR-regulated mammalian xenobiotic response.

\section{Acknowledgments}

We thank Joyce Barwick and Philip Guzelian for hepatocyte preparation and transfection and Alex Shearer for assistance in the TetRE-VP-CAR transgene construction.

\section{References}

Chen LJ and Segel IH (1985) Purification and characterization of bile salt sulfotransferase from human liver. Arch Biochem Biophys 241:371-379.

Falany JL and Falany CN (1996) Expression of cytosolic sulfotransferases in normal mammary epithelial cells and breast cancer cell lines. Cancer Res 56:1551-1555. Fisher MM, Magnusson R, and Miyai K (1971) Bile acid metabolism in mammals. I. Bile acid induced intrahepatic cholestasis. Lab Investig 25:88-91.

Garcia-Allan C, Lord PG, Loughlin JM, Orton TC, and Sidaway JE (2000) Identification of phenobarbitone-modulated genes in mouse liver by differential display. $J$ Biochem Mol Toxicol 14:65-72.

Glatt H (1997) Sulfation and sulfotransferases 4: bioactivation of mutagens via sulfation. FASEB J 11:314-321.

Goodwin B, Jones SA, Price RR, Watson MA, McKee DD, Moore LB, Galardi C, Wilson JG, Lewis MC, Roth ME, et al. (2000) A regulatory cascade of the nuclear receptors FXR, SHP-1, and LRH-1 represses bile acid biosynthesis. Mol Cell 6:517-526.

Goodwin B, Moore LB, Stoltz CM, McKee DD, and Kliewer SA (2001) Regulation of the human CYP2B6 gene by the nuclear pregnane X receptor. Mol Pharmaco 60:427-431.

Honkakoski P, Zelko I, Sueyoshi T, and Negishi M (1998) The nuclear orphan receptor CAR-retinoid $\mathrm{X}$ receptor heterodimer activates the phenobarbitalresponsive enhancer module of the CYP2B gene. Mol Cell Biol 18:5652-5658.

Huang W, Zhang J, Chua SS, Qatanani M, Han Y, Granata R, and Moore DD (2003) Induction of bilirubin clearance by the constitutive androstane receptor (CAR) Proc Natl Acad Sci USA 100:4156-4161.

Kast HR, Goodwin B, Tarr PT, Jones SA, Anisfeld AM, Stoltz CM, Tontonoz P, Kliewer SA, Willson TM, and Edwards PA (2002) Regulation of multidrug resistance-associated protein $2(\mathrm{ABCC} 2)$ by the nuclear receptors pregnane $\mathrm{X}$ receptor, farnesoid X-activated receptor and constitutive androstane receptor. J Biol Chem 277:2908-2915.

Kistner A, Gossen M, Zimmermann F, Jerecic J, Ullmer C, Lubbert H, and Bujard H (1996) Doxycycline-mediated quantitative and tissue-specific control of gene expression in transgenic mice. Proc Natl Acad Sci USA 93:10933-10938.

Kitada H, Miyata M, Nakamura T, Tozawa A, Honma W, Shimada M, Nagata K Sinal CJ, Guo GL, Gonzalez FJ, et al. (2003) Protective role of hydroxysteroid sulfotransferase in lithocholic acid-induced liver toxicity. J Biol Chem 278:1783817844 .

Klaassen CD, Liu L, and Dunn RT 2nd (1998) Regulation of sulfotransferase mRNA expression in male and female rats of various ages. Chem Biol Interact 109:299 313.

Kullak-Ublick GA, Stieger B, Hagenbuch B, and Meier PJ (2000) Hepatic transpor of bile salts. Semin Liver Dis 20:273-292.

Leuschner U, Czygan P, Liersch M, Frohling W, and Stiehl A (1977) Morphologic studies on the toxicity of sulfated and nonsulfated lithocholic acid in the isolationperfused rat liver. $Z$ Gastroenterol 15:246-253.

Lu TT, Makishima M, Repa JJ, Schoonjans K, Kerr TA, Auwerx J, Mangelsdorf DJ (2000) Molecular basis for feedback regulation of bile acid synthesis by nuclear receptors. Mol Cell 6:507-515.

Lyle S, Stanczak J, Ng K, and Schwartz NB (1994) Rat chondrosarcoma ATP sulfurylase and adenosine 5 '-phosphosulfate kinase reside on a single bifunctional protein. Biochemistry 33:5920-5925.

Maglich JM, Stoltz CM, Goodwin B, Hawkins-Brown D, Moore JT, and Kliewer SA (2002) Nuclear pregnane X receptor and constitutive androstane receptor regulate overlapping but distinct sets of genes involved in xenobiotic detoxification. Mol Pharmacol 62:638-646. 
Makishima M, Lu TT, Xie W, Whitfield GK, Domoto H, Evans RM, Haussler MR, and Mangelsdorf DJ (2002) Vitamin D receptor as an intestinal bile acid sensor. Science (Wash DC) 296:1313-1316.

Makishima M, Okamoto AY, Repa JJ, Tu H, Learned RM, Luk A, Hull MV, Lustig $\mathrm{KD}$, Mangelsdorf DJ, and Shan B (1999) Identification of a nuclear receptor for bile acids. Science (Wash DC) 284:1362-1365.

Narisawa T, Magadia NE, Weisburger JH, and Wynder EL (1974) Promoting effect of bile acids on colon carcinogenesis after intrarectal instillation of $N$-methyl- $N^{\prime}$ nitro- $N$-nitrosoguanidine in rats. J Natl Cancer Inst 53:1093-1097.

Parks DJ, Blanchard SG, Bledsoe RK, Chandra G, Consler TG, Kliewer SA, Stimme JB, Willson TM, Zavacki AM, et al. (1999) Bile acids: natural ligands for an orphan nuclear receptor. Science (Wash DC) 284:1365-1368.

Radominska A, Comer KA, Zimniak P, Falany J, Iscan M, and Falany CN (1990) Human liver steroid sulphotransferase sulphates bile acids. Biochem $J$ 272:597604

Rosenfeld JM, Vargus Jr R, Xie W, and Evans RM (2003) Genetic versus chemical profiling definition of a xenobiotic gene network controlled by the nuclear receptor PXR. Mol Endocrinol 17:1268-1282.

Runge-Morris M, Wu W, and Kocarek TA (1999) Regulation of rat hepatic hydroxysteroid sulfotransferase (SULT2-40/41) gene expression by glucocorticoids: evidence for a dual mechanism of transcriptional control. Mol Pharmacol 56:11981206.

Song CS, Echchgadda I, Baek BS, Ahn SC, Oh T, Roy AK, and Chatterjee B (2001) Dehydroepiandrosterone sulfotransferase gene induction by bile acid activated farnesoid X receptor. J Biol Chem 276:42549-42556.

Sonoda J, Xie W, Rosenfeld JM, Barwick JL, Guzelian PS, and Evans RM (2002) Regulation of a sulfonation cascade by nuclear receptor PXR. Proc Natl Acad Sci USA 99:13801-13806.

Staudinger JL, Goodwin B, Jones SA, Hawkins-Brown D, MacKenzie KI, LaTour A Liu Y, Klaassen CD, Brown KK, Reinhard J, et al. (2001) The nuclear receptor PXR is a lithocholic acid sensor that protects against liver toxicity. Proc Natl Acad Sci USA 98:3369-3374.

Sugatani J, Kojima H, Ueda A, Kakizaki S, Yoshinari K, Gong QH, Owens IS, Negishi M, and Sueyoshi T (2001) The phenobarbital response enhancer module in the human bilirubin UDP- glucuronosyltransferase UGT1A1 gene and regulation by the nuclear receptor CAR. Hepatology 33:1232-1238.

Tzameli I, Pissios P, Schuetz EG, and Moore DD (2000) The xenobiotic compound
1,4-Bis[2-(3,5-dichloropyridyloxy)]benzene is an agonist ligand for the nuclear receptor CAR. Mol Cell Biol 20:2951-2958.

Ueda A, Hamadeh HK, Webb HK, Yamamoto Y, Sueyoshi T, Afshari CA, Lehmann JM, and Negishi M (2002) Diverse roles of the nuclear orphan receptor CAR in regulating hepatic genes in response to phenobarbital. Mol Pharmacol 61:1-6.

Wang H, Chen J, Hollister K, Sowers LC, Forman BM (1999) Endogenous bile acids are ligands for the nuclear receptor FXR/BAR. Mol Cell 3:543-553.

Wei P, Zhang J, Egan-Hafley M, Liang S, and Moore DD (2000) The nuclear receptor CAR mediates specific xenobiotic induction of drug metabolism. Nature (Lond) 407:920-923.

Wei P, Zhang J, Dowhan DH, Han Y, and Moore DD (2002) Specific and overlapping functions of the nuclear hormone receptors CAR and PXR in xenobiotic response. Pharmacogenomics $J$ 2:117-126.

Xie W, Chow LT, Paterson AJ, Chin E, and Kudlow JE (1999) Conditional expression of erbB2 oncogene in transgenic mice elicits striking hyperplasia in stratified epithelia and up-regulation of TGF $\alpha$ expression. Oncogene 18:3593-3607.

Xie W, Barwick JL, Simon CM, Pierce A, Safe S, Blumberg B, Guzelian PS, and Evans RM (2000a) Reciprocal activation of xenobiotic response genes by nuclear receptors SXR/PXR and CAR. Genes Dev 14:3014-3023.

Xie W, Barwick JL, Downes M, Blumberg B, Simon CM, Nelson MC, NeuschwanderTetri BA, Brunt EM, Guzelian PS, and Evans RM (2000b) Humanized xenobiotic response in mice expressing nuclear receptor SXR. Nature (Lond) 406:435-439.

Xie W, Radominska-Pandya A, Shi Y, Simon CM, Nelson MC, Ong ES, Waxman DJ, and Evans RM (2001) An essential role for nuclear receptors SXR/PXR in detoxification of cholestatic bile acids. Proc Natl Acad Sci USA 98:3375-3380.

Xie W, Yeuh M-F, Radominska-Pandya A, Saini SPS, Nigishi Y, Bottroff BS, Cabrera GY, Tukey RH, and Evans RM (2003) Control of steroid, heme and carcinogen metabolism by nuclear pregnane $\mathrm{X}$ receptor and constitutive androstane receptor Proc Natl Acad Sci USA 100:4150-4155.

Zhang J, Huang W, Chua SS, Wei P, and Moore DD (2002) Modulation of acetaminophen-induced hepatotoxicity by the xenobiotic receptor CAR. Science (Wash DC) 298:422-424.

Address correspondence to: Wen Xie, Center for Pharmacogenetics, Salk Hall 656, University of Pittsburgh, Pittsburgh, PA 15213. E-mail: wex6@pitt.edu 